\title{
Engineering Hydrophobic Organosilica Nanoparticle-Doped Nanofibers for Enhanced and Fouling Resistant Membrane Distillation
}

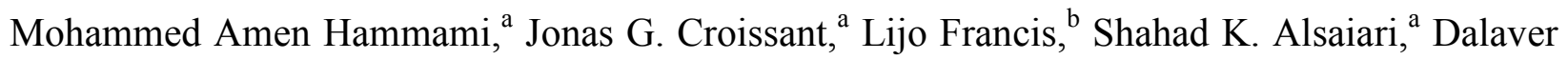 \\ H. Anjum, ${ }^{\mathrm{c}}$ Noreddine Ghaffour, ${ }^{\mathrm{b}}$ and Niveen M. Khashab. ${ }^{*, \mathrm{a}}$ \\ ${ }^{a}$ Smart Hybrid Materials Laboratory, Advanced Membranes and Porous Materials Center, King Abdullah \\ University of Science and Technology (KAUST), Thuwal 23955-6900, Saudi Arabia. \\ ${ }^{\mathrm{b}}$ Water Desalination and Reuse Center, KAUST, Thuwal 23955-6900, Saudi Arabia. \\ ${ }^{\mathrm{c}}$ Imaging and Characterization Core Laboratory, KAUST, Thuwal 23955-6900, Saudi Arabia.
}

\begin{abstract}
Engineering and scaling-up new materials for better water desalination are imperative to find alternative fresh water sources to meet future demands. Herein, the fabrication of hydrophobic polyetherimide composite nanofiber membranes doped with novel ethylenepentafluorophenylene-based periodic mesoporous organosilica nanoparticles is reported for enhanced and fouling resistant membrane distillation. Novel organosilica nanoparticles were homogeneously incorporated into electrospun nanofiber membranes depicting a proportional increase of hydrophobicity to the particle contents. Direct contact membrane distillation experiments on the organosilica-doped membrane with only $5 \%$ doping showed an increase of flux of $140 \%$ compared to commercial membranes. The high porosity of organosilica nanoparticles was further utilized to load the eugenol antimicrobial agent which produced a dramatic enhancement of the anti-biofouling properties of the membrane of $70 \%$ after $24 \mathrm{~h}$.
\end{abstract}

Keywords: nanofibers, periodic mesoporous organosilica, organosilica nanoparticles, biofouling, membrane distillation. 


\section{INTRODUCTION}

Fresh water shortage is considered one of the most critical problems nowadays. The majority of the world population lacks drinking water according to the world health organization (WHO). ${ }^{1}$ Seawater is widely available and composes the majority of the world resources. The growing importance of water desalination encouraged the renewed focus on existing technology for water desalination. Reverse osmosis (RO) and multistage flash (MSF) are the major and most commonly used technologies for seawater desalination. ${ }^{2}$ However, the evolutionary improvement of RO membranes solely prepared from polymeric materials seems to be approaching saturation especially pertaining to improving flux and reducing energy consumption. ${ }^{3-5}$ Furthermore, biofouling is a key limitation of commercial RO membranes which require extensive pretreatment and excessive pressure to overcome the resistance arising from fouled membranes. ${ }^{4,6-7}$

To overcome these limitations, researchers sought methods with lower capital and operational expenditures such as membrane distillation (MD), which is a thermally driven membrane based water desalination process. MD can produce high purity water without hydraulic pressure and it runs at low temperatures which make it a relatively low-cost desalination process. ${ }^{8-9}$ Although MD is a promising technology, one of the challenges facing its development is the fabrication of efficient membranes with specific characteristics such as high hydrophobicity, optimum pore size, high porosity, and low thermal conductivity. ${ }^{10-12}$ Furthermore, biofouling is also one of the major challenges for MD and has a critical impact since it decreases the MD performances by reducing the membrane flux and lifetime or by deteriorating the product water quality due to increased pore wetting and blockage. ${ }^{13-15}$ The conventional phase inversion technique has been mainly used for MD membrane preparation. 
However, membranes derived from this technique have several limitations in terms of permeate flux performance due to their low porosity. The membrane hydrophobicity and liquid entry pressure (LEP) are critical parameters in MD, which reduce membrane pore wetting during the process.

Cost-effective membranes are characterized by high hydrophobicity to generate a selfcleaning effect and reduce the adhesion of bacteria. ${ }^{16-17}$ To enhance the surface hydrophobicity, researchers reported the use of materials with low surface energy ${ }^{18}$ or increased surface roughness. ${ }^{19}$ Other techniques used for improving hydrophobicity included self-assembly, ${ }^{20-21}$ chemical vapor deposition ${ }^{22}$ template synthesis, ${ }^{23}$ chemical modification $^{24}$ and more recently electrospinning. ${ }^{25}$ Electrospinning has been used to fabricate highly porous and hydrophobic nanofiber membranes. Compared with the traditional MD membranes, the electrospun nanofiber membranes (ENM) promise more interesting advantages for MD application to obtain higher permeate flux. Since Feng et $a l .^{26}$ first explored the polyvinylidene fluoride (PVDF) nanofiber for MD and claimed over $99 \%$ of salt rejection and a competitive permeate flux, several studies have been done on exploring PVDF and modified PVDF nanofibers for MD applications to enhance the permeate flux and stability. ${ }^{27-30}$ Few studies have been done using polymers other than PVDF including Matrimid $^{\circledR},{ }^{31}$ polyoxadiazoles $^{24}$ and poly(trimethyl hexamethylene terephthalamide). ${ }^{32}$ Polyetherimide (PEI) is a commercially available and cost effective polymer, but MD applications were only reported on PEI flat sheet membranes. ${ }^{33-34}$

Incorporating NPs into electrospun nanofibers or hollow fibers has been recently heavily pursued to impart functionalities to membranes. ${ }^{35-38}$ Metallic NPs such as silver and gold have been employed as antimicrobial agents to reduce biofouling of membranes. ${ }^{39-42}$ Silica NPs were also successfully used to improve the hydrophobicity and the lifetime of membranes. ${ }^{43-46}$ The 
major practical challenges for the incorporation of NPs into membranes are the high cost, poor reproducibility, and difficulty in scaling up nano-membrane manufacturing processes for commercial use. Furthermore, health and safety issues around the use of nanomaterials have to be addressed in the domestic water industry, particularly with respect to the use of NPs.

In this work, a facile and scalable fabrication of composite nanofiber membrane for simultaneously enhanced MD and anti-biofouling is described. The engineered system comprised PEI nanofiber doped with novel ethylene-pentafluorophenylene bridged periodic mesoporous organosilica (PMO) NPs to enhance the hydrophobicity of the composite membrane (Scheme 1). PMO NPs are hybrid nano-objects with very high organic content $(\sim 30-70 \mathrm{wt} \%)$ and have thus higher properties than organically-doped silica NPs which have lower organic content $\left(\sim 1-30 \mathrm{wt}^{\mathrm{O}} \%\right)^{47-50}$ Although scalable and relatively cheap to produce, PMO NPs differ from conventional mesoporous silica by their tunable hydrophilicity/hydrophobicity along with many other properties that are associated with the type of organic bridges chosen for the design. ${ }^{51-54}$ The PMO doped nanofiber (PEI-PMO NPs) showed resistance to bacterial attachment mainly due to the increased surface roughness and low surface energy. Loading PMO NPs with the eugenol antioxidant/antimicrobial agent ${ }^{55}$ boosted the antibiofouling properties by $70 \%$ after 24 $\mathrm{h}$, as the nanofiber membranes released antimicrobial agents in the presence of bacteria (Scheme 1). This fouling resistant composite membrane showed excellent thermal properties with more than $140 \%$ flux increase. This is the first report on PMO-doped PEI nanofibers composite membranes that are superior to the commercially available PTFE membranes in flux and antibiofouling enhancement. 
A

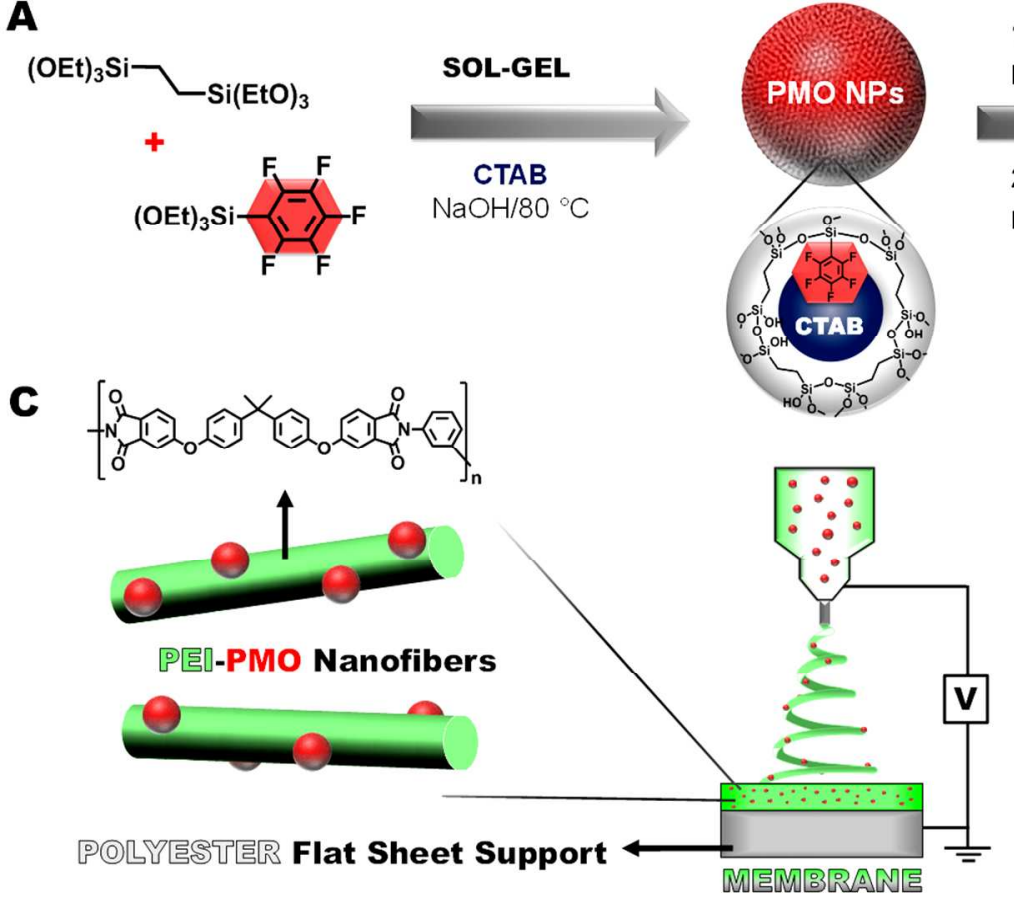

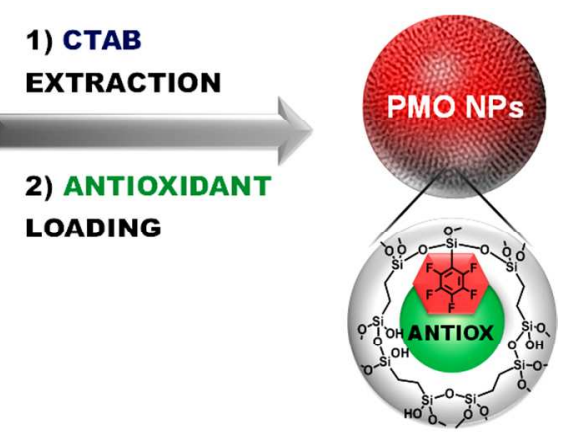

B

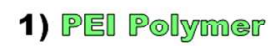

NMP, $25^{\circ} \mathrm{C}$

2) ELECTROSPINNING

Scheme 1. Representation of the design and antioxidant/antimicrobial loading of PMO NPs (A) for the preparation of PEI-PMO NPs feed solution (B) for electrospinning PMO-doped PEI nanofiber membrane $(\mathrm{C})$.

\section{EXPERIMENTAL SECTION}

2.1. Materials. Commercial polyetherimide (PEI) of molecular weight of $55000 \mathrm{~g} \mathrm{~mol}^{-1}$ was supplied by SABIC Innovation Plastics under the trade name of Grade PEI ULTEM ${ }^{\mathrm{TM}}$ 1000P. NMethyl-2-pyrrolidone (NMP), cetyltrimethylammonium bromide, sodium hydroxide, ammonium nitrate, bis(triethoxysilyl)ethane and (pentafluorophenyl)triethoxysilane were purchased from Sigma-Aldrich. The polyester nonwoven support was purchased from Hirose Paper Mfg Co Ltd Japan. The commercial PTFE membrane was utilized to compare the performance of the seawater desalination with the functional PEI nanofibrous membranes presented in this study. 
2.2. Preparation of PMO NPs. A mixture of CTAB (500 mg, $1.37 \mathrm{mmol})$, distilled water (240 $\mathrm{mL})$, and sodium hydroxide $(1.75 \mathrm{~mL}, 2 \mathrm{M})$ was stirred at $80^{\circ} \mathrm{C}$ for $1 \mathrm{~h}$ at $700 \mathrm{rpm}$ in a $500 \mathrm{~mL}$ three neck round bottom flask. The sol-gel process was then started by a quick addition of bis(triethoxysilyl)ethane $(600 \mu \mathrm{L}, 1.62 \mathrm{mmol})$ and (pentafluorophenyl)triethoxysilane $(200 \mu \mathrm{L}$, $0.75 \mathrm{mmol})$. The solution was stirred at $1000 \mathrm{rpm}$ at $80^{\circ} \mathrm{C}$ for $3 \mathrm{~h}$. Afterward, the solution was cooled to room temperature while stirring; fractions were gathered in propylene tubes and collected by centrifugation during 15 minutes at $21 \mathrm{krpm}$. The as-prepared sample was sonicated twice with an alcoholic solution of ammonium nitrate $\left(6 \mathrm{~g} \mathrm{~L}^{-1}\right)$ at $45{ }^{\circ} \mathrm{C}$ for 30 minutes and washed three times with ethanol, water, and ethanol. Each washing was followed by centrifugation collection in propylene tubes during 15 minutes at $21 \mathrm{krpm}$. The as-prepared CTAB-free PMO NPs were dried under vacuum for few hours.

2.3. Preparation of the PEI nanofiber membranes. The PEI polymer was dissolved in the NMP solvent (15 to $17 \mathrm{wt} \%$ ) under magnetic stirring at $50{ }^{\circ} \mathrm{C}$ for $24 \mathrm{~h}$. A fraction of this PEI solution $(6 \mathrm{~mL})$ was electrospun at $25^{\circ} \mathrm{C}$ using a $10 \mathrm{~mL}$ syringe with a "21 Gauge" needle at a mass flow rate of $1 \mathrm{~mL} \mathrm{~h}^{-1}$. A high voltage, $(20 \mathrm{kV})$ was applied between the needle and the plate collector, and an automatic system was used to move the collector in a zig-zag direction to obtain a uniform membrane. The nanofibers were collected on the highly porous polyester support mounted on the grounded collector plate and the thickness of the membrane was controlled by experimental time.

\subsection{Preparation of PEI-PMO nanofiber membranes. In order to make the} nanocomposite membranes (Scheme 1), PMO (5 or $10 \mathrm{wt} \%$ of the polymer weight) was dispersed in (NMP) followed by ultrasonication and then PEI was added up to $17 \mathrm{wt} \%$ to $20 \mathrm{wt} \%$ 
of the total weight and dissolved by using a magnetic stirrer at $50{ }^{\circ} \mathrm{C}$ for $24 \mathrm{~h}$. A fraction of this PEI-PMO solution (6 mL) was electrospun at $25^{\circ} \mathrm{C}$ using a $10 \mathrm{~mL}$ syringe with a "21 Gauge" needle at a mass flow rate of $1 \mathrm{~mL} \mathrm{~h}^{-1}$. The experiment was then carried out in the manner as that of PEI nanofiber membranes.

2.5. Apparatus and methods. The surface morphologies of the PMO NPs and the electrospun nanofibrous membrane were investigated by field emission scanning electron microscopy (FE-SEM) using SEM Quanta 600 and Nova Nano 630 FEG. For SEM analysis nanofibers were collected on aluminum foil. Prior to SEM analysis, the electrospun membranes were sputter coated with iridium. The average fiber diameter was measured from the SEM image using an image analyzer Image $\mathrm{J}$ software. HRTEM was obtained using Titan ST (FEI Company) operating at $300 \mathrm{kV}$ and equipped with a $4 \times 4 \mathrm{k}$ CCD camera (Gatan). Standard (TEM) images were collected using a Tecnai G2 Spirit TWIN 20-120 kV/LaB6. The PMO NPs were dispersed in ethanol and drop cast on a lacy copper grid and dried for $1 \mathrm{~h}$ prior to the analysis. The electrospun nanofibers were directly spun on the copper and subjected to TEM imaging. The chemical composition of the PMO NP was measured by FTIR spectrometer in the range of 500 to $4000 \mathrm{~cm}^{-1}$. The X-ray powder diffraction (XRD) patterns were obtained for PMOs by a Bruker D8 Advance at a scanning rate of 2 deg $\min ^{-1}$ in the $2 \theta$ range of 2 to 80 degrees. XPS analysis was utilized to analyze the surface composition of the PMO NPs and the electrospun nanofiber.The nanofiber membrane topography was measured by an (AFM) using Agilent 5400 SPM instrument (USA). The decomposition behavior of the composites was studied using a thermogravimetric analyzer (TGA) (TG 209 F1 Iris, Netzsch, Germany) under nitrogen from 30 to $1000{ }^{\circ} \mathrm{C}$ at a ramping rate of $10 \mathrm{deg} \mathrm{min}^{-1}$. The wettability of the membrane 
was determined by water contact angle technique (WCA). The membrane porosity was measured by a mercury porosimetry. Sorption-desorption analyses were performed with a Micromeritics ASAP 2420 instrument.

2.6. Desalination performance using DCMD. All the membranes were cut into $7 \times 12$ $\mathrm{cm}$ pieces to fit into the DCMD module. Seawater was preheated to the desired temperature (45, 55,65 and $75^{\circ} \mathrm{C}$ ) and circulated through the active side of the membrane, whereas the pure cold water was maintained at $20^{\circ} \mathrm{C}$ (coolant) and circulated through the other side of the membrane. The salt concentration of both feed and coolant were determined by a conductivity meter (OaktonEutech Instruments, Malaysia).

\subsection{Antioxidant/antimicrobial agent loading and release procedure. PMO NPs $(25$} $\mathrm{mg})$ were dispersed in ethanol solution. Then, eugenol $(100 \mathrm{mg})$ was added to the solution and stirred overnight. After centrifugation, the supernatant was dried in a vacuum oven. Eugenol release from the PMOs and the nanofiber were conducted by placing PMO NPs (2 mg) and nanofiber $(20 \mathrm{mg})$ in deionized water and sea water. At predetermined time interval, the solution was centrifugated and $3 \mathrm{~mL}$ were taken from the solution and studied by UV-VIS spectrophotometer (Varian Cary 5000) at $282 \mathrm{~nm}$ for eugenol.

2.8. Adhesion assay. One isolated colony of the E. coli Top 10 (Invitrogen) transformed with a plasmid giving an ampicillin resistance was put in culture in LB media containing ampicillin $\left(50 \mu \mathrm{g} \mathrm{mL}{ }^{-1}\right)$ at $37^{\circ} \mathrm{C}$. The commercial PTFE, PEI, PEI-PMOs and cargo-loaded PEIPMOs nanofibers specimen were incubated with $1 \mathrm{~mL}$ of the culture $\left(\mathrm{OD}_{600 \mathrm{~nm}}=0.6-0.8\right)$ for $1 \mathrm{~h}$ 
and $24 \mathrm{~h}$ and washed 3 times with PBS. The different membranes were then put in culture in LB media containing ampicillin at $37{ }^{\circ} \mathrm{C}$ overnight. A serial dilution of the obtained cultures was performed and used to spread on the surfaces LB-agar plates containing ampicillin to evaluate the CFU mL $\mathrm{mL}^{-1}$.

2.9. Inhibition growth assay. One isolated colony of the E. coli Top 10 (Invitrogen) transformed with a plasmid giving an ampicillin resistance was put in culture in LB media containing ampicillin at $37{ }^{\circ} \mathrm{C}$ overnight. A fraction of this culture $(100 \mu \mathrm{L})$ was spread on the surface of LB-agar plates containing ampicillin and the commercial PTFE, PEI, PEI/PMOs and cargo-loaded PEI/PMOs nanofibers specimen were placed on the surface of the plates in triplicates and incubated at $37^{\circ} \mathrm{C}$ overnight.

\section{RESULTS AND DISCUSSION}

3.1. Fabrication and characterization of PMO NPs. A templated aqueous solution of cetyltrimethylammonium bromide with sodium hydroxide was stirred at $80{ }^{\circ} \mathrm{C}$ for $1 \mathrm{~h}$ followed by a quick addition of bis(triethoxysilyl)ethane and (pentafluorophenyl)triethoxysilane to start a $3 \mathrm{~h}$ sol-gel process (Scheme 1A). The particles were then surfactant-extracted, vacuumed-dried and then characterized. Transmission electron microscopy (TEM) analysis of PMO NPs showed uniformly distributed non-aggregated spherical nano-objects of 100 to $200 \mathrm{~nm}$ with cubicordered porosity (Figures 1A, S1, and 2E). X-ray diffraction (XRD) at low and wide-angle confirmed the mesoporosity by the intense peak at $1.8 \mathrm{deg}$ and the amorphous nature of the siloxane network with the broad peak from 16 to $35 \mathrm{deg}$, respectively (Figure S2). Based on the 
sorption analysis presented hereafter yielding $772 \mathrm{~m}^{2} \mathrm{~g}^{-1}$ of surface area, pore sizes of ca. $2.9 \mathrm{~nm}$ and the pore to pore distance of $4.9 \mathrm{~nm}$ determined by the Bragg's law, the corresponding PMO pore thickness averages $2 \mathrm{~nm}$. The FTIR spectrum of PMO NPs confirmed the high condensation degree of the siloxane network with the $v_{\text {Si-O }}$ in the 1100 to $1040 \mathrm{~cm}^{-1}$ region as well as the incorporation of the ethane and pentafluorophenylene moieties with the $v_{\mathrm{C}-\mathrm{H}}$ aliphatic around 2990 $\mathrm{cm}^{-1}$ and the $\delta_{\mathrm{Csp2-F}}$ from 790 to $680 \mathrm{~cm}^{-1}$ (Figure S3). The presence of silicon, oxygen, carbon, and fluorine atoms in PMO NPs was further demonstrated by elemental mapping coupled with electron microscopy (see Figure 2A).

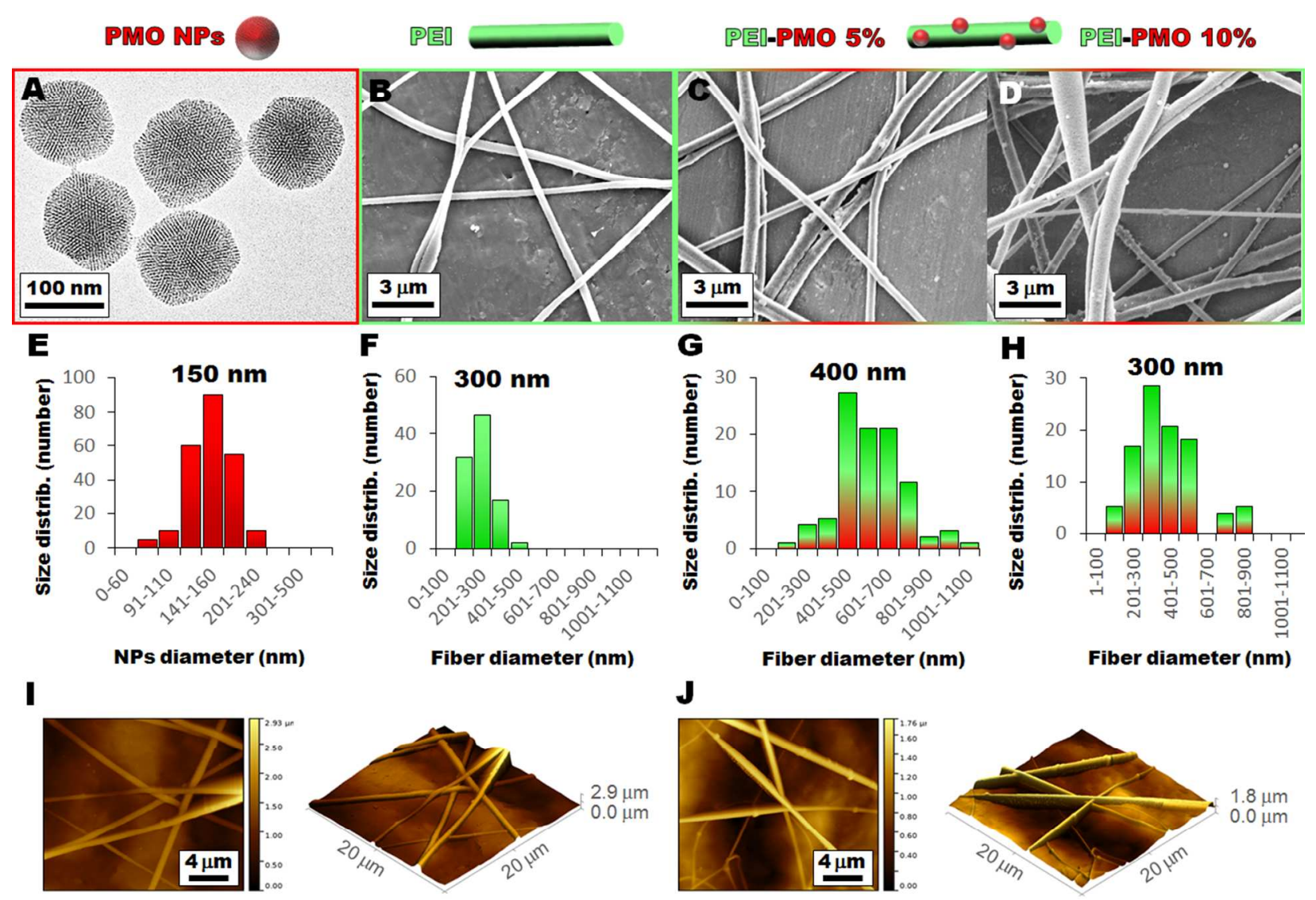

Figure 1. TEM micrographs of PMO NPs (A). SEM micrographs of PEI (B), and PEI-PMO nanofibers with $5 \%(\mathrm{C})$, and $10 \%$ (D), of PMO NPs. Statistical analyses of the diameter distributions of PMO NPs (E), PEI (F), PEI-PMO nanofibers with $5 \%$ loading (G), and PEI- 
PMO nanofibers with $10 \%$ loading $(\mathrm{H})$, of PMO NPs. AFM micrographs of PEI-PMO nanofibers with $5 \%$ (I), and $10 \%(\mathrm{~J})$, of PMO NPs.

\subsection{Fabrication and characterization of PMO-doped nanofiber membrane. In}

order to study the electrospinnabilty of the bare PEI polymer, we initially tested three different concentrations of PEI polymer into the NMP solvent. At low concentration $(15 \mathrm{wt} \%)$, the chain overlap was not sufficient and beaded fibers were observed by SEM (Figure S4A). Upon increases of the concentration to $17 \mathrm{wt} \%$, the better macromolecule entanglement allowed a satisfactory control of the electrospinning jet producing smooth beads-free PEI nanofibers (Figure S4B).

The doping of fluorinated PMO NPs into PEI nanofibers was then carried out and investigated with various techniques. The incorporation was successfully performed with different particle contents as displayed by SEM micrographs depicting the surface morphologies of the pristine PEI nanofibers and of the PEI/PMOs with $5 \%$ and $10 \%$ of PMO NPs (Figure 1BD). The surfaces of both the pristine and the composite nanofibers were smooth and uniform. Relatively narrow diameter distributions were calculated via the Image J software for PMO NPs and for the various nanofibers (Figure 1E-H). The doping of the particles increased the diameter distribution of the nanofibers. This phenomenon can be explained by the increase of the dope solution viscosity. The viscosity was measured at $25{ }^{\circ} \mathrm{C}$ and indeed increased from $818 \mathrm{mPa} \cdot \mathrm{s}$ without particles to 948 and $1115 \mathrm{mPa}$ s with 5 and $10 \mathrm{wt} \%$ of PMO particle dopings, respectively (Table S1). Atomic force microscopy (AFM) micrographs confirmed that the PMO NPs were attached to the surface of the nanofibers (Figure 1I-J). AFM also qualitatively suggested an increase of the surface roughness of the nanofiber membranes. TEM and scanning transmission electron microscopy (STEM) further confirmed the uniform dispersion of PMO NPs in the PEI nanofiber (Figure 2B-E). The PMO NPs appeared to be very well intercalated 
within the fiber matrix (see Figures 2B-E and S4C). Using both 5 and $10 \mathrm{wt} \%$ of particle concentration, homogenous PEI-PMO nanofibers could be obtained (Figure S5), though PMO concentrations higher than $5 \mathrm{wt} \%$ generated increasing amount of particle aggregations (e.g. severe aggregation at $20 \mathrm{wt} \%$, data not shown). Note that the application of a high electric potential to the dope solution during the electrospinning process causing the ultrafast ejection of the dope solution, accounts for to the orientation of the majority of NPs on the surface of nanofibers. Using spatially-resolved energy-dispersive spectroscopy (EDS) on a segment of particle-free nanofibers and on a grafted particle ( 1 and 2, respectively in Figure 2D), the incorporation of PMO NPs onto PEI nanofibers was demonstrated (Figure 2F-G). This assertion was confirmed on a large area of the membranes via x-ray photoelectron spectroscopy (XPS) measurements (Figure S6). The XPS spectrum of the pristine PEI nanofiber reflected the polymer composition, while the incorporation of PMO NPs generated the appearance of Si atoms (Figure S6C-D). In addition, by increasing the loading amount of particles from 5 to $10 \mathrm{wt} \%$, the ratio between the nitrogen over silicon ratio $(\mathrm{Si} / \mathrm{N})$ consistently increased in the membranes. 

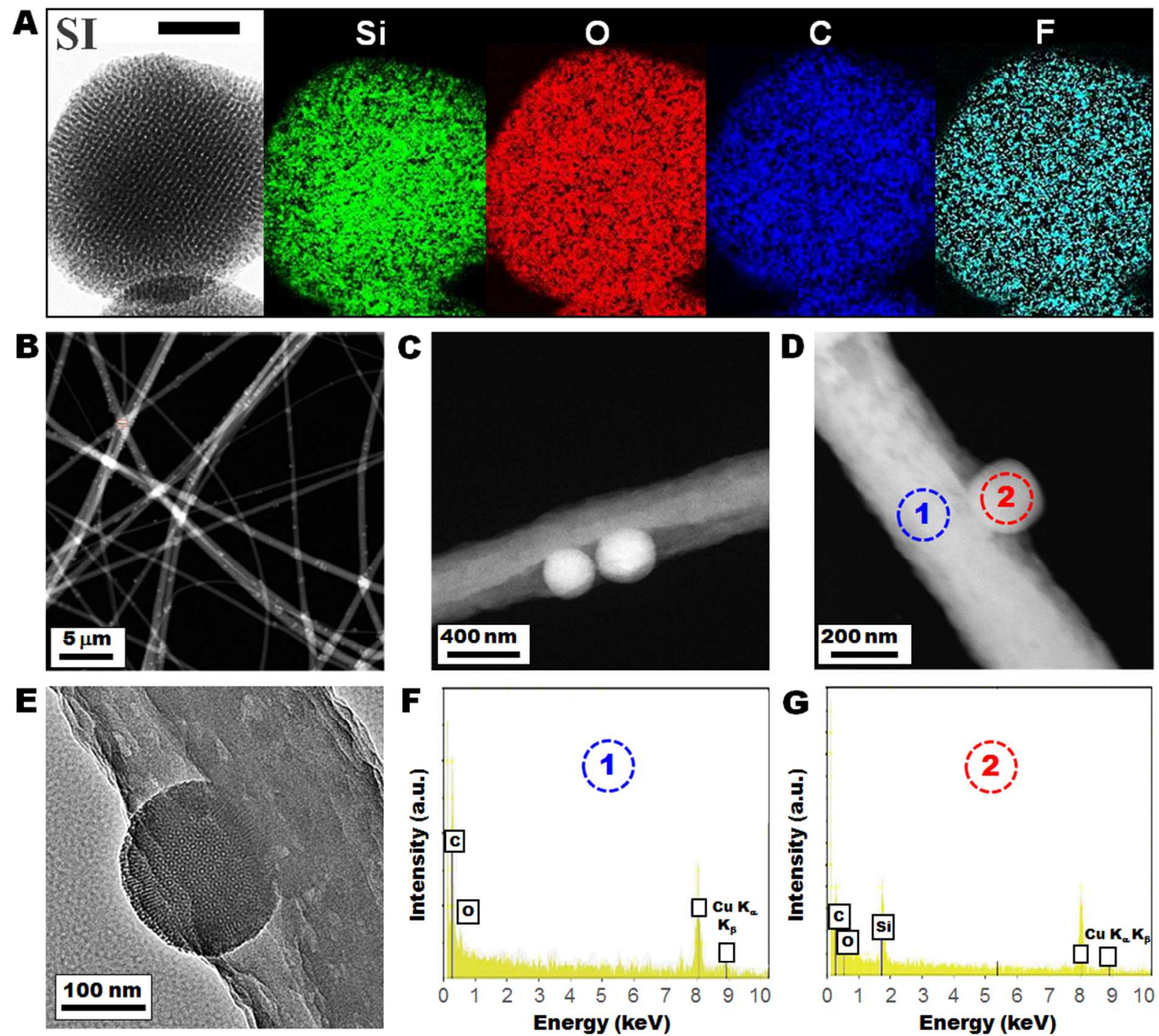

Figure 2. STEM-EELS analysis of PMO NPs (A). Representative spectrum image (SI) of NPs with the silicon, oxygen, carbon, and fluorine elemental mappings. STEM (B-D) and TEM (E) images of PEI-PMO nanofibers with $10 \%$ of PMO NPs. EDS spectra of PEI-PMO nanofibers with $10 \%$ of PMO NPs acquired on the PEI nanofiber (F) and the PMO NP (G) grafted onto it, see numbers 1 and 2, respectively on the corresponding STEM image (D).

The evaluation of other physico-chemical properties of the membrane was then performed. The thermal stability of membrane was first studied by thermogravimetric analysis (TGA) studies. TGA analyses were performed on PEI fibers, PMO NPs, and on PEI/PMO composite nanofibers (Figure S7). All membranes showed a first weight loss $(<5 \mathrm{wt} \%)$ from 100 to $200{ }^{\circ} \mathrm{C}$ 
corresponding to solvent evaporation and a second weight loss from ca. 500 to $700{ }^{\circ} \mathrm{C}$ corresponding to the degradation of the polymer. TGA thus showed that PMOs NPs did not affect significantly the thermal properties of the composite membranes. The hydrophobicity of the membrane was then studied via water contact angle (WCA) measurements. First, the electrospun PEI membrane showed a higher hydrophobicity than that of the PEI membranes obtained via the casting technique, as shown by the important increase of the WCA from 64 to 123 degrees (Figure 3). This was attributed to the rough topology associated with the nanofiber structure. Besides, experimental results showed an increase of the WCA from 123 to 142 degrees by increasing the percent of PMO NPs doping from 5 to $10 \mathrm{wt} \%$ due to the hydrophobic ethylene-pentafluorophenylene-based particle composition (Figure 3). Previous studies ${ }^{56-58}$ combined with the increase of liquid entry pressures (Table S2) suggested that the increase of the WCA was also correlated to the enhanced roughness associated with the presence of PMO NPs. The porosity of the membrane was then analyzed the by sorption analyses. Using nitrogen sorption and the Brunauer-Emmett-Teller (BET) theory was used to calculate surface areas of PMO NPs and of the different membranes. The PMO NPs showed a typical type IV isotherm characteristic of mesoporous materials (Figure 4A), the BET surface area was of $772 \mathrm{~m}^{2} \mathrm{~g}^{-1}$ and the BJH (Barrett-Joyner-Halenda) pore size distribution was centered at $2.5 \mathrm{~nm}$ (Figure 4E). The pristine PEI nanofiber exhibited a BET surface area of $68.7 \mathrm{~m}^{2} \mathrm{~g}^{-1}$, and after the addition of 5 and $10 \mathrm{wt} \%$ of PMO NPs the surface area rose to $70.1 \mathrm{~m}^{2} \mathrm{~g}^{-1}$ and $78.1 \mathrm{~m}^{2} \mathrm{~g}^{-1}$, respectively. A wide distribution was noticed for the pristine PEI nanofiber from $5 \mathrm{~nm}$ to $65 \mathrm{~nm}$, and the pore size corresponding to the PMO NPs appeared in the analysis of the PEI-PMO samples. Mercury porosimetry was also performed to study the effect of membrane thickness on the macroporosity of the system. All ENMs with different thicknesses and the composite PEI-PMO ENM displayed 
high porosities on the order of 77 to $86 \%$ with mean pore sizes at around one micron (Figure S8).

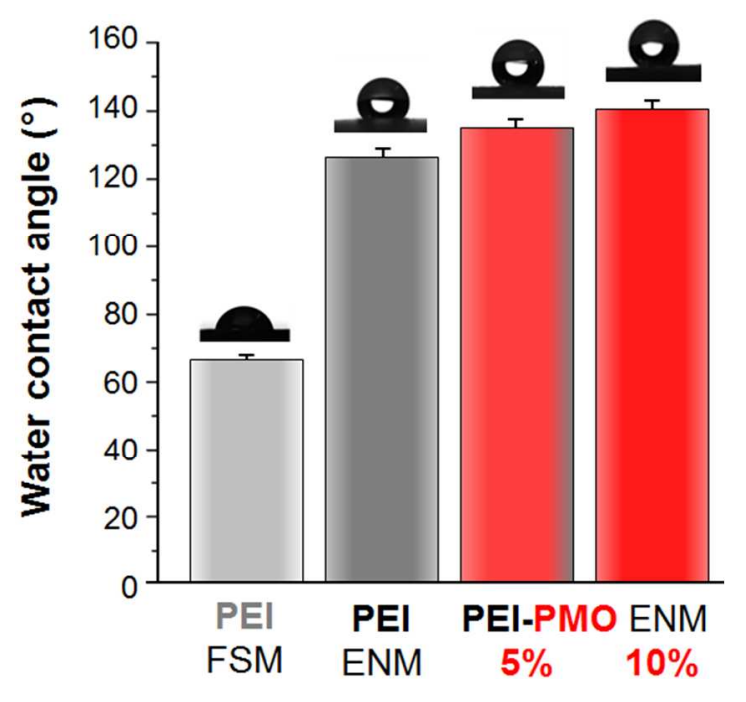

Figure 3. Water contact angle measurements of PEI flat sheet membrane (FSM), PEI and PEIPMO electrospun nanofiber membranes (ENM). 

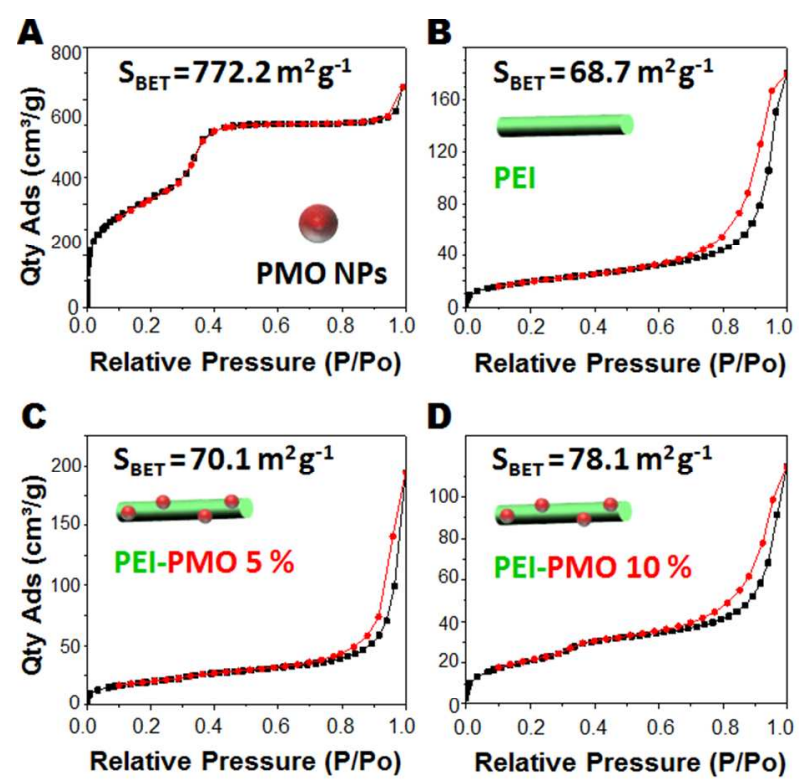

\section{D}

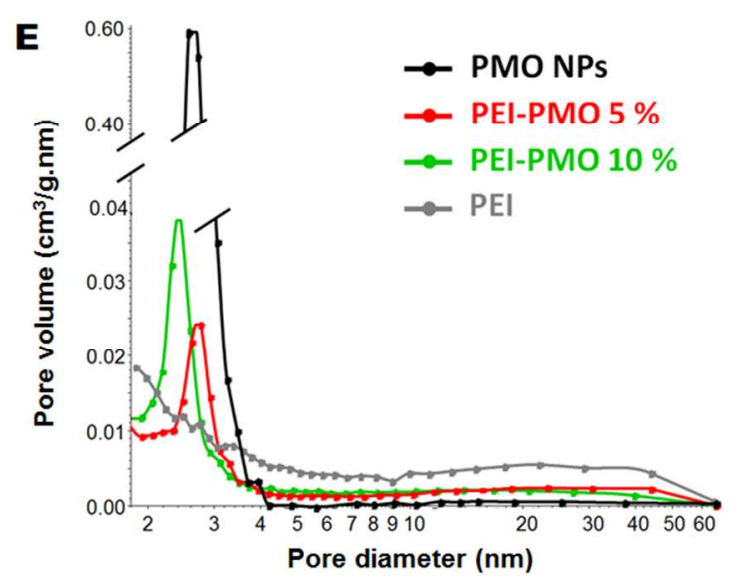

Figure 4. Nitrogen sorption isotherms of PMO NPs (A), PEI (B), and PEI-PMO $5 \%$ (C) and $10 \%$ (D) of particles. BJH pore distribution of PMO NPs, PEI and PEI-PMO ENM (E).

3.3. Membrane distillation studies. The engineered PEI-PMO composite ENM showed the desired characteristics to be employed as a suitable membrane for MD. Figure 5 shows the water vapor flux data observed during direct contact membrane distillation (DCMD) process using different electrospun membranes and one commercial polytetrafluoroethylene (PTFE) membrane (see properties in Table S3). The results were compared with the pristine PEI ENM and the commercial PTFE membrane at different feed inlet temperatures and under the same 
operating conditions. Red Sea water was used as the feed solution for all experiments. Different membranes showed vapor flux productions at different rates and the observed trend was as follows: PEI-PMO (5 \%) ENM > PEI-PMO (10 \%) ENM > PEI ENM > commercial PTFE. For example, at a $65{ }^{\circ} \mathrm{C}$ feed inlet temperature, the pristine PEI ENM showed more than a $100 \%$ increase in the water vapor flux compared to the commercial PTFE membrane. Furthermore, under the same operating conditions, PEI-PMO (5\%) ENM and PEI-PMO (10\%) ENM showed 140 and $130 \%$ increases in the vapor flux compared to the commercial PTFE membrane, respectively (Figure 5). The increase of the hydrophobicity of the PEI-PMO ENM at the surface compared to the PEI ENM could have provided more resistance to water penetration and the partial pore blockage to ensure higher flux compared to the pure PEI ENM. The minor fraction of PMO aggregates for PEI-PMO with $10 \mathrm{wt} \%$ of particle doping may have been the reason for the lower performance compared to the PEI-PMO $5 \mathrm{wt} \%$. All the experimental results were reproduced three times and error bars were within the acceptable range (Figure 5). SEM analyses of the PEI-PMO membrane after MD applications for $24 \mathrm{~h}$ confirmed the stability of the PMO particle incorporation (Figure S9). 
PEI ENM

PEI-PMO 10\% ENM

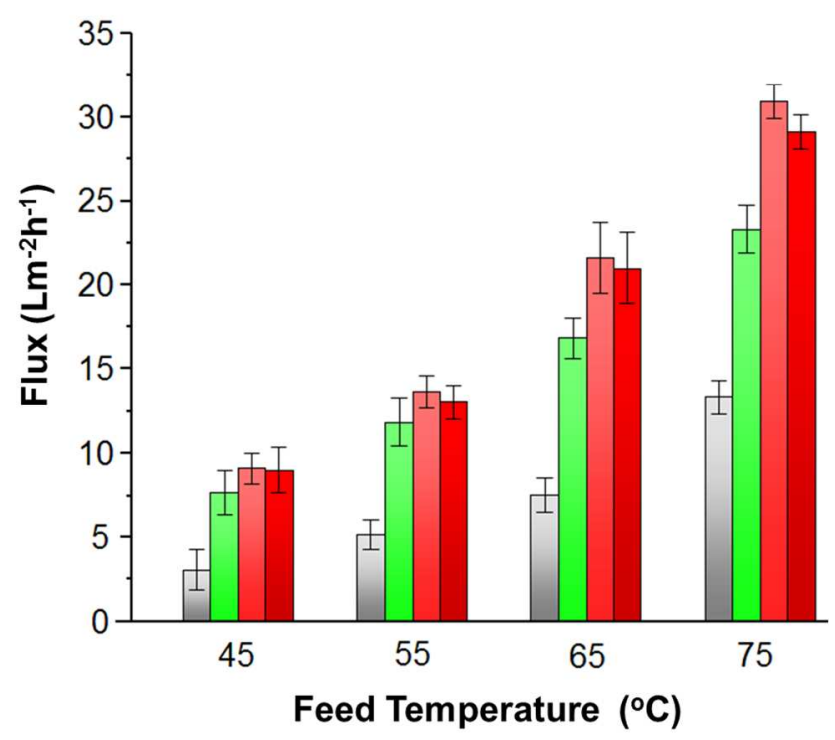

Figure 5. Direct contact MD flux as a function of the feed temperature (coolant temperature $20^{\circ} \mathrm{C}$ ) for the commercial membrane, PEI and PEI-PMO ENM. The membrane thickness is 60 $\mu \mathrm{m}$.

3.4. Biofouling studies. To evaluate the bacterial adhesion as well as the potential biofilm formation, ampicillin resistant E. coli strains were used (see ESI). PEI, PEI-PMO and eugenolloaded PEI-PMO ENMs were incubated in LB cultures of ampicillin resistant E. coli. The membranes were then put in contact with the surfaces of LB-agar plates containing ampicillin. The counting of the colony forming units (CFUs) was performed in order to evaluate the bacterial adhesion onto the membranes. As shown in Figure 6, the adhesion of E. coli was significantly reduced (around $40 \%$ ) by using PEI-PMOs versus the pristine PEI nanofiber. In addition, higher anti-biofouling properties could be obtained with PEI-PMO membranes with PMO NPs loaded with the eugenol antimicrobial agent, which yielded a reduction of $70 \%$ in the bacterial attachment after $24 \mathrm{~h}$ of bacterial exposure. 


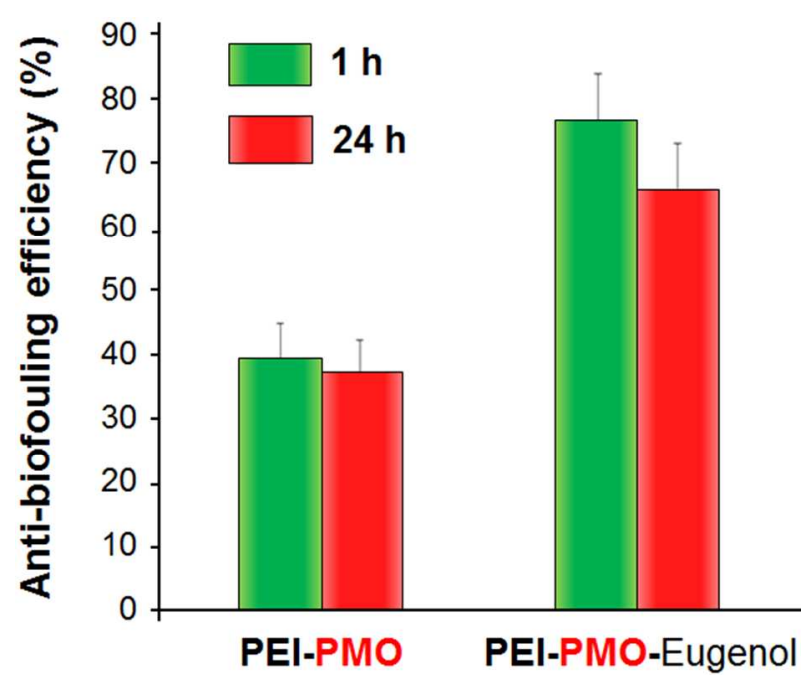

Figure 6. Comparison of the anti-biofouling efficiencies of PEI-PMO and PEI-PMO-Eugenol. The biofouling corresponds to the percentage reduction of the bacterial attachment compared to the PEI ENM.

The antibacterial activity of the eugenol-loaded composite membranes was further evaluated using ampicillin resistant E. coli Top 10 bacteria strain to detect only the added bacteria. The conditions used promoted a rapid bacterial growth on surfaces. Ampicillin resistant E. coli cultures were spread on the surface of LB-agar plates containing ampicillin and PEI-PMO or eugenol-loaded PEI-PMO ENMs. No bacterial killing was detected in the case of PEI-PMO ENM, however, in the case of eugenol-loaded PEI-PMO ENM a clear bacterial killing was observed directly after adding the composite membrane (Figure 7). Note that, eugenol is insoluble in seawater, hence no release was detected over 60 days of testing, which ensured the long-term anti-microbial activity of the composite membranes. This result demonstrated that eugenol-loaded PEI-PMO membrane was able to effectively inhibit the bacterial growth which is promising for long-term MD. 


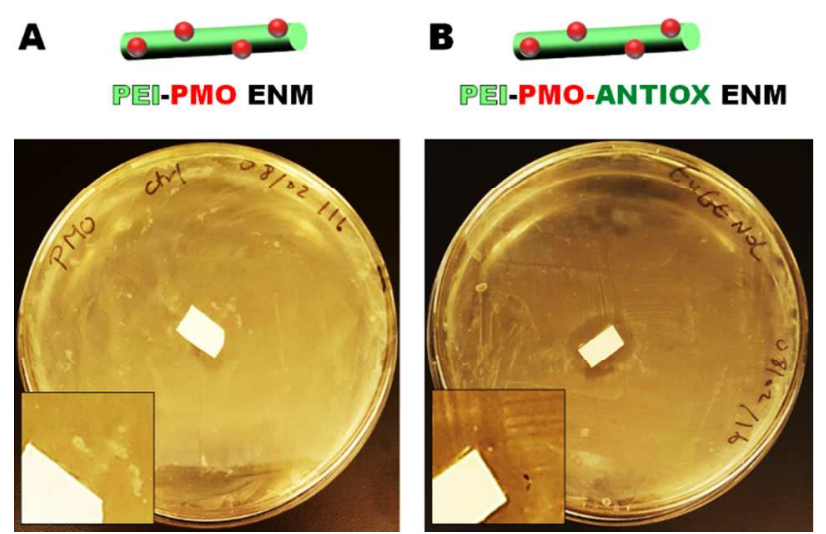

Figure 7. Biofouling experiment with PEI-PMO (A) and PEI-PMO loaded with Eugenol (B) demonstrating the controlled release property imparted to the membrane through the use of porous PMO.

\section{CONCLUSIONS}

In summary, novel ethylene-pentafluorophenylene-based periodic mesoporous organosilica nanoparticles were doped into PEI composite nanofiber membranes for enhanced and fouling resistant membrane distillation. The organosilica particles possessed hydrophobic ethane and pentafluorophenyl moieties which enhanced the hydrophobicity of the membrane and allowed the loading of antimicrobial and antioxidant agents. The resulting nanofiber membrane depicted a high porosity of up to $84 \%$ with a homogeneous and stable incorporation of the nanoparticles as shown by multiple techniques. The composite membranes were successfully employed for the production of fresh water from the Red Sea. The prepared membranes showed enhanced water vapor production as high as $31 \mathrm{~L} \mathrm{~m}^{2} \mathrm{~h}^{-1}$ with a $140 \%$ increase compared to the commercial PTFE flat sheet membrane. Furthermore, a dramatic decrease of $70 \%$ of the bacterial attachment was observed via antimicrobial agent-loaded nanoparticles doped into the membrane. The facile and scalable fabrication of these nanofiber membranes coupled the potential of environmentfriendly mesoporous silica-based nanocontainers have great potential to provide innovative 
mixed-matrix membranes that can drastically improve industrially valuable processes such as membrane distillation.

\section{- AUTHOR INFORMATION}

\section{Corresponding Author}

*E-mail: Niveen.khashab@kaust.edu.sa. Tel: +966-28021172. Fax: +966-28082410.

\section{Author Contributions}

All authors contributed to the work.

\section{Notes}

The authors declare no competing financial interest.

\section{- ACKNOWLEDGMENTS}

We gratefully acknowledge support from KAUST.

\section{- ASSOCIATED CONTENT}

\section{*Supporting Information}

The Supporting Information is available free of charge on the ACS Publications website at DOI: am-2016-11167k. Supplementary experimental sections, additional characterization data of the PMOs, PEI and PMO-PEI ENM. TEM and SEM characterization data; XRD and FTIR spectra; and TGA and XPS analyses.

\section{- REFERENCES}

1. Progress on Drinking-Water and Sanitation-2012 Update, Launched on 6 March 2012. Comprehensive Assessment of the Freshwater Resources of the World, World Health Organization (WHO) 2012. 
2. Shannon, M. A.; Bohn, P. W.; Elimelech, M.; Georgiadis, J. G.; Mariñas, B. J.; Mayes, A. M. Science and Technology for Water Purification in the Coming Decades. Nature 2008, 452, 301-310.

3. Elimelech, M.; Phillip, W. A. The Future of Seawater Desalination: Energy, Technology, and the Environment. science 2011, 333, 712-717.

4. Ghaffour, N.; Missimer, T. M.; Amy, G. L. Technical Review and Evaluation of the Economics of Water Desalination: Current and Future Challenges for Better Water Supply Sustainability. Desalination 2013, 309, 197-207.

5. Amy, G.; Ghaffour, N.; Li, Z.; Francis, L.; Linares, R. V.; Missimer, T.; Lattemann, S. MembraneBased Seawater Desalination: Present and Future Prospects. Desalination 2016, 401, 16-21.

6. Lee, K. P.; Arnot, T. C.; Mattia, D. A Review of Reverse Osmosis Membrane Materials for Desalination-Development to Date and Future Potential. J. Membr. Sci. 2011, 370, 1-22.

7. Kim, S. J.; Ko, S. H.; Kang, K. H.; Han, J. Direct Seawater Desalination by lon Concentration Polarization. Nat. Nanotechnol. 2010, 5, 297-301.

8. Ding, Z.; Liu, L.; El-Bourawi, M. S.; Ma, R. Analysis of a Solar-Powered Membrane Distillation System. Desalination 2005, 172, 27-40.

9. $\quad$ El-Bourawi, M.; Ding, Z.; Ma, R.; Khayet, M. A Framework for Better Understanding Membrane Distillation Separation Process. J. Membr. Sci. 2006, 285, 4-29.

10. Khayet, M.; Cojocaru, C.; García-Payo, M. d. C. Experimental Design and Optimization of Asymmetric Flat-Sheet Membranes Prepared for Direct Contact Membrane Distillation. J. Membr. Sci. 2010, 351, 234-245.

11. Lafuma, A.; Quéré, D. Superhydrophobic States. Nat. Mater. 2003, 2, 457-460.

12. Cardea, S.; Gugliuzza, A.; Sessa, M.; Aceto, M.; Drioli, E.; Reverchon, E. Supercritical Gel Drying: A Powerful Tool for Tailoring Symmetric Porous PVDF-HFP Membranes. ACS Appl. Mater. Interfaces 2009, 1, 171-180.

13. Zodrow, K. R.; Bar-Zeev, E.; Giannetto, M. J.; Elimelech, M. Biofouling and Microbial Communities in Membrane Distillation and Reverse Osmosis. Environ. Sci. Technol. 2014, 48, 1315513164.

14. Goh, S.; Zhang, J.; Liu, Y.; Fane, A. G. Fouling and Wetting in Membrane Distillation (MD) and MD-Bioreactor (MDBR) for Wastewater Reclamation. Desalination 2013, 323, 39-47.

15. Tijing, L. D.; Woo, Y. C.; Choi, J.-S.; Lee, S.; Kim, S.-H.; Shon, H. K. Fouling and its Control in Membrane Distillation-a Review. J. Membr. Sci. 2015, 475, 215-244.

16. Privett, B. J.; Youn, J.; Hong, S. A.; Lee, J.; Han, J.; Shin, J. H.; Schoenfisch, M. H. Antibacterial Fluorinated Silica Colloid Superhydrophobic Surfaces. Langmuir 2011, 27, 9597-9601.

17. Marmur, A. Underwater Superhydrophobicity: Theoretical Feasibility. Langmuir 2006, 22, 14001402.

18. Feng, L.; Li, S.; Li, H.; Zhai, J.; Song, Y.; Jiang, L.; Zhu, D. Super-Hydrophobic Surface of Aligned Polyacrylonitrile Nanofibers. Angew. Chem. Int. Ed. 2002, 114, 1269-1271.

19. Ji, J.; Fu, J.; Shen, J. Fabrication of a Superhydrophobic Surface from the Amplified Exponential Growth of a Multilayer. Adv. Mater. 2006, 18, 1441-1444.

20. Lai, W.-C.; Cheng, L.-T. Preparation and Characterization of Novel Poly (Vinylidene Fluoride) Membranes Using Self-Assembled Dibenzylidene Sorbitol for Membrane Distillation. Desalination 2014, 332, 7-17.

21. Lin, N.-J.; Yang, H.-S.; Chang, Y.; Tung, K.-L.; Chen, W.-H.; Cheng, H.-W.; Hsiao, S.-W.; Aimar, P.; Yamamoto, K.; Lai, J.-Y. Surface Self-Assembled Pegylation of Fluoro-Based PVDF Membranes via Hydrophobic-Driven Copolymer Anchoring for Ultra-Stable Biofouling Resistance. Langmuir 2013, 29 , 10183-10193.

22. Asatekin, A.; Gleason, K. K. Polymeric Nanopore Membranes for Hydrophobicity-Based Separations by Conformal Initiated Chemical Vapor Deposition. Nano Lett. 2010, 11, 677-686. 
23. Sun, M.; Luo, C.; Xu, L.; Ji, H.; Ouyang, Q.; Yu, D.; Chen, Y. Artificial Lotus Leaf by Nanocasting. Langmuir 2005, 21, 8978-8981.

24. Maab, H.; Francis, L.; Al-Saadi, A.; Aubry, C.; Ghaffour, N.; Amy, G.; Nunes, S. P. Synthesis and Fabrication of Nanostructured Hydrophobic Polyazole Membranes for Low-Energy Water Recovery. J. Membr. Sci. 2012, 423, 11-19.

25. Jiang, L.; Zhao, Y.; Zhai, J. A Lotus-Leaf-Like Superhydrophobic Surface: A Porous Microsphere/Nanofiber Composite Film Prepared by Electrohydrodynamics. Angew. Chem. Int. Ed. 2004, 116, 4438-4441.

26. Feng, C.; Khulbe, K.; Matsuura, T.; Gopal, R.; Kaur, S.; Ramakrishna, S.; Khayet, M. Production of Drinking Water from Saline Water by Air-Gap Membrane Distillation Using Polyvinylidene Fluoride Nanofiber Membrane. J. Membr. Sci. 2008, 311, 1-6.

27. Francis, L.; Ghaffour, N.; Alsaadi, A. S.; Nunes, S. P.; Amy, G. L. PVDF Hollow Fiber and Nanofiber Membranes for Fresh Water Reclamation Using Membrane Distillation. J. Mater. Sci. 2014, 49, 20452053.

28. Liao, Y.; Wang, R.; Tian, M.; Qiu, C.; Fane, A. G. Fabrication of Polyvinylidene Fluoride (PVDF) Nanofiber Membranes by Electro-Spinning for Direct Contact Membrane Distillation. J. Membr. Sci. 2013, 425, 30-39.

29. Lalia, B. S.; Guillen, E.; Arafat, H. A.; Hashaikeh, R. Nanocrystalline Cellulose Reinforced PVDFHFP Membranes for Membrane Distillation Application. Desalination 2014, 332, 134-141.

30. Dong, Z.-Q.; Ma, X.-h.; Xu, Z.-L.; You, W.-T.; Li, F.-b. Superhydrophobic PVDF-PTFE Electrospun Nanofibrous Membranes for Desalination by Vacuum Membrane Distillation. Desalination 2014, 347, 175-183.

31. Francis, L.; Maab, H.; AlSaadi, A.; Nunes, S.; Ghaffour, N.; Amy, G. Fabrication of Electrospun Nanofibrous Membranes for Membrane Distillation Application. Desalination and Water Treatment 2013, 51, 1337-1343.

32. Guo, F.; Servi, A.; Liu, A.; Gleason, K. K.; Rutledge, G. C. Desalination by Membrane Distillation Using Electrospun Polyamide Fiber Membranes with Surface Fluorination by Chemical Vapor Deposition. ACS Appl. Mater. Interfaces 2015, 7, 8225-8232.

33. Khayet, M.; Matsuura, T. Application of Surface Modifying Macromolecules for the Preparation of Membranes for Membrane Distillation. Desalination 2003, 158, 51-56.

34. Khayet, M.; Mengual, J.; Matsuura, T. Porous Hydrophobic/Hydrophilic Composite Membranes: Application in Desalination Using Direct Contact Membrane Distillation. J. Membr. Sci. 2005, 252, 101113.

35. Sharma, N.; McKeown, S. J.; Ma, X.; Pochan, D. J.; Cloutier, S. G. Structure- Property Correlations in Hybrid Polymer- Nanoparticle Electrospun Fibers and Plasmonic Control over their Dichroic Behavior. ACS Nano 2010, 4, 5551-5558.

36. Puigmartí-Luis, J.; Pérez del Pino, Á.; Laukhina, E.; Esquena, J.; Laukhin, V.; Rovira, C.; VidalGancedo, J.; Kanaras, A. G.; Nichols, R. J.; Brust, M. Shaping Supramolecular Nanofibers with Nanoparticles Forming Complementary Hydrogen Bonds. Angew. Chem. Int. Ed. 2008, 120, 1887-1891. 37. Han, E.; Wu, D.; Qi, S.; Tian, G.; Niu, H.; Shang, G.; Yan, X.; Yang, X. Incorporation of Silver Nanoparticles into the Bulk of the Electrospun Ultrafine Polyimide Nanofibers via a Direct lon Exchange Self-Metallization Process. ACS Appl. Mater. Interfaces 2012, 4, 2583-2590.

38. Mitschang, F.; Schmalz, H.; Agarwal, S.; Greiner, A. Tea-Bag-Like Polymer Nanoreactors Filled with Gold Nanoparticles. Angew. Chem. Int. Ed. 2014, 53, 4972-4975.

39. Koga, H.; Kitaoka, T.; Wariishi, H. In Situ Synthesis of Silver Nanoparticles on Zinc Oxide Whiskers Incorporated in a Paper Matrix for Antibacterial Applications. J. Mater. Chem. 2009, 19, 2135-2140. 
40. Hsu, A.; Liu, F.; Leung, Y. H.; Ma, A. P.; Djurišić, A. B.; Leung, F. C.; Chan, W. K.; Lee, H. K. Is the Effect of Surface Modifying Molecules on Antibacterial Activity Universal for a Given Material? Nanoscale 2014, 6, 10323-10331.

41. Li, Y.; Zhang, W.; Niu, J.; Chen, Y. Mechanism of Photogenerated Reactive Oxygen Species and Correlation with the Antibacterial Properties of Engineered Metal-Oxide Nanoparticles. ACS Nano 2012, 6, 5164-5173.

42. Faupel, F.; Zaporojtchenko, V.; Strunskus, T.; Elbahri, M. Metal-Polymer Nanocomposites for Functional Applications. Adv. Eng. Mater. 2010, 12, 1177-1190.

43. Zhang, Y.; Wang, R. Fabrication of Novel Polyetherimide-Fluorinated Silica Organic-Inorganic Composite Hollow Fiber Membranes Intended for Membrane Contactor Application. J. Membr. Sci. 2013, 443, 170-180.

44. Wu, H.; Mansouri, J.; Chen, V. Silica Nanoparticles as Carriers of Antifouling Ligands for PVDF Ultrafiltration Membranes. J. Membr. Sci. 2013, 433, 135-151.

45. Lee, J.; Boo, C.; Ryu, W.-H.; Taylor, A. D.; Elimelech, M. Development of Omniphobic Desalination Membranes Using a Charged Electrospun Nanofiber Scaffold. ACS Appl. Mater. Interfaces 2016, 8, 11154-11161.

46. Boo, C.; Lee, J.; Elimelech, M. Engineering Surface Energy and Nanostructure of Microporous Films for Expanded Membrane Distillation Applications. Environ. Sci. Technol. 2016, 50, 8112-8119. 47. Croissant, J. G.; Cattoën, X.; Wong Chi Man, M.; Durand, J.-O.; Khashab, N. M. Syntheses and Applications of Periodic Mesoporous Organosilica Nanoparticles. Nanoscale 2015, 7, 20318-20334.

48. Croissant, J. G.; Picard, S.; Aggad, D.; Klausen, M.; Mauriello-Jimenez, C.; Maynadier, M.; Mongin, O.; Clermont, G.; Genin, E.; Cattoen, X.; Wong Chi Man, M.; Raehm, L.; Garcia, M.; Gary-Bobo, M.; Blanchard-Desce, M.; Durand, J.-O. Fluorescent Periodic Mesoporous Organosilica Nanoparticles Dual-Functionalized via Click Chemistry for Two-Photon Photodynamic Therapy in Cells. J. Mater. Chem. B 2016, 4, 5567-5574.

49. Jimenez, C. M.; Knezevic, N. Z.; Rubio, Y. G.; Szunerits, S.; Boukherroub, R.; Teodorescu, F.; Croissant, J. G.; Hocine, O.; Seric, M.; Raehm, L.; Stojanovic, V.; Aggad, D.; Maynadier, M.; Garcia, M.; Gary-Bobo, M.; Durand, J.-O. Nanodiamond-PMO for Two-Photon Pdt and Drug Delivery. J. Mater. Chem. B 2016, 4, 5803-5808.

50. Fatieiev, Y.; Croissant, J.; Julfakyan, K.; Deng, L.; Anjum, D. H.; Gurinov, A.; Khashab, N. Enzymatically Degradable Hybrid Organic-Inorganic Bridged Silsesquioxane Nanoparticles for In Vitro Imaging. Nanoscale 2015, 7, 15046-15050.

51. Croissant, J.; Cattoën, X.; Man, M. W. C.; Gallud, A.; Raehm, L.; Trens, P.; Maynadier, M.; Durand, J. O. Biodegradable Ethylene-Bis (Propyl) Disulfide-Based Periodic Mesoporous Organosilica Nanorods and Nanospheres for Efficient In-Vitro Drug Delivery. Adv. Mater. 2014, 26, 6174-6180.

52. Croissant, J.; Cattoën, X.; Wong Chi Man, M.; Dieudonné, P.; Charnay, C.; Raehm, L.; Durand, J. O. One-Pot Construction of Multipodal Hybrid Periodic Mesoporous Organosilica Nanoparticles with Crystal-Like Architectures. Adv. Mater. 2015, 27, 145-149.

53. Waki, M.; Maegawa, Y.; Hara, K.; Goto, Y.; Shirai, S.; Yamada, Y.; Mizoshita, N.; Tani, T.; Chun, W.-J.; Muratsugu, S.; Tada, M.; Fukuoka, A.; Inagaki, S. A Solid Chelating Ligand: Periodic Mesoporous Organosilica Containing 2,2'-Bipyridine within the Pore Walls. J. Am. Chem. Soc. 2014, 136, 4003-4011. 54. Chen, Y.; Shi, J. Chemistry of Mesoporous Organosilica in Nanotechnology: Molecularly OrganicInorganic Hybridization into Frameworks. Adv. Mater. 2016, 28, 3235-3272.

55. Carbone-Howell, A. L.; Stebbins, N. D.; Uhrich, K. E. Poly (Anhydride-Esters) Comprised Exclusively of Naturally Occurring Antimicrobials and EDTA: Antioxidant and Antibacterial Activities. Biomacromolecules 2014, 15, 1889-1895.

56. Dalvi, V. H.; Rossky, P. J. Molecular Origins of Fluorocarbon Hydrophobicity. Proc. Natl. Acad. Sci. 2010, 107, 13603-13607. 
57. Tijing, L. D.; Woo, Y. C.; Shim, W.-G.; He, T.; Choi, J.-S.; Kim, S.-H.; Shon, H. K. Superhydrophobic Nanofiber Membrane Containing Carbon Nanotubes for High-Performance Direct Contact Membrane Distillation. J. Membr. Sci. 2016, 502, 158-170.

58. Edwie, F.; Teoh, M. M.; Chung, T.-S. Effects of Additives on Dual-Layer Hydrophobic-Hydrophilic Pvdf Hollow Fiber Membranes for Membrane Distillation and Continuous Performance. Chem. Eng. Sci. 2012, 68, 567-578. 


\section{TOC GRAPHIC}

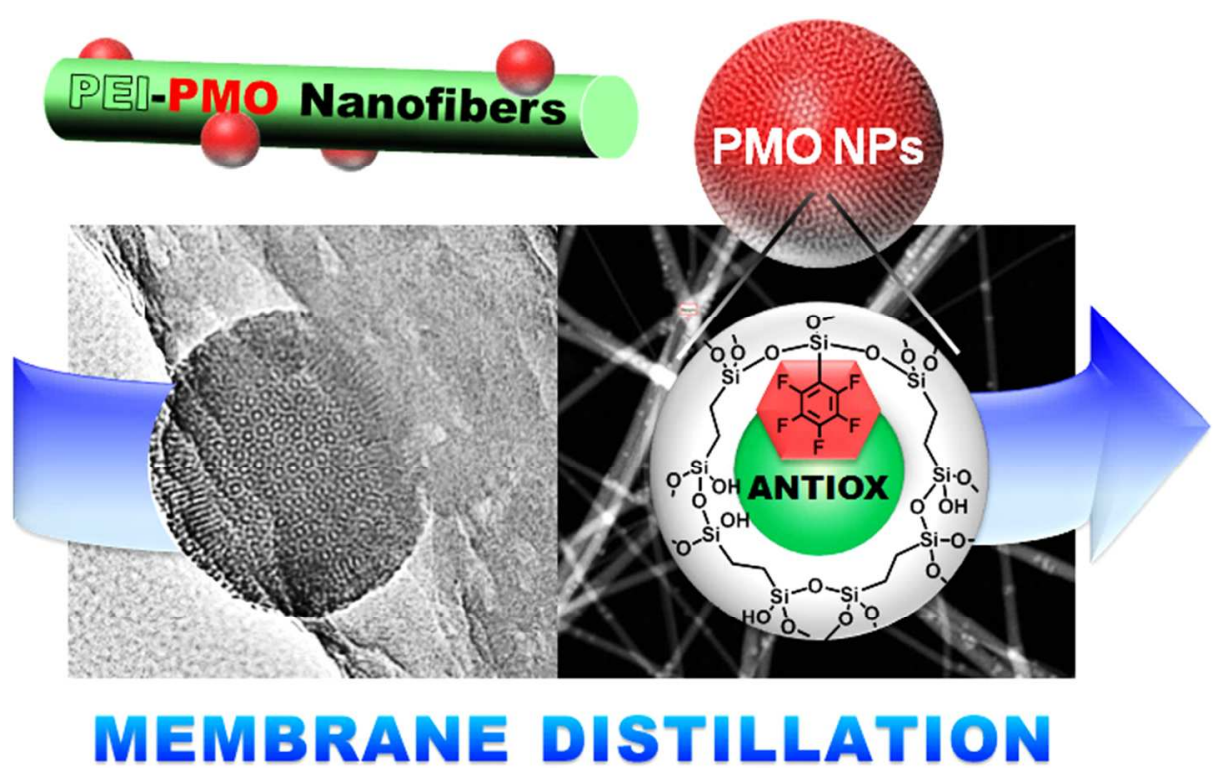

